\title{
Analyse comparée du chargement horizontal de deux quais sur pieux en service à partir d'instrumentations
}

\author{
Humberto Yáñez-Godoy*1, Franck Schoefs*2
}

* Université de Nantes, Institut de Recherche en Génie Civil et Mécanique (GeM), UMR 6183 CNRS, Faculté des Sciences et des Techniques, 2 rue de la Houssinière, Nantes, F-44000 France.

\author{
IDoctorant; humberto.yanez@univ-nantes.fr \\ ${ }^{2}$ Maître de conférences ; franck.schoefs@univ-nantes.fr
}

\section{Résumé}

La modélisation du comportement mécanique global ou de certains éléments des structures portuaires comme les quais, lors de leur construction ou de leur mise en service, est d'un intérêt primordial en vue de leur suivi, de l'expertise des pathologies ultérieures et de leur requalification. Cet article se focalise sur la comparaison des efforts mesurés dans les tirants passifs de deux quais sur pieux, identiques dans leur conception, construits au début des années 2000. Les instrumentations permettent d'analyser le comportement de l'ouvrage sous chargement horizontal dans son ensemble par l'étude des variations des efforts le long du quai. L'évolution des efforts dans le temps ainsi que de l'effet de la marée sont étudiés.

Mots-clés : quais sur pieux, instrumentation de quais, tirants d'ancrage

\begin{abstract}
Modelling of the mechanical behaviour of harbour structures such as on-pile quays, either locally or globally, during the construction stages and/or during their service life, is of prime importance for the evaluation of structural pathologies and for their re-analysis. This paper focuses on the comparison of the loading in anchoring tie-rods for two on-piles quays, identical in their conception, built during early 2000. The data acquired from the installed instrumentations allow the analysis of quay behaviour under horizontal loading by analysing the variations of the measured loading along the structure. The temporal evolution of the loading as well as the effect of the tides are also studied.
\end{abstract}

Key words: on-piles quays, instrumentation, tie-rods

\section{Introduction}

Le respect des règles de l'Art considéré lors de l'exécution d'un projet conditionne pour une grande part la durabilité d'un ouvrage. La connaissance d'événements observés ou prédits pendant la construction et l'exploitation d'un ouvrage permet une optimisation du suivi des structures qui conduit à une amélioration des programmes d'inspection, de maintenance et de réparation. Plus généralement la requalification de ces structures nécessite la compréhension de leur comportement réel. L'instrumentation peut s'avérer d'une aide importante en complément des modélisations usuelles qu'elles soient au niveau du comportement mécanique ou 
du chargement, en particulier si des problèmes couplés difficiles apparaissent ou bien si des conditions de réalisation risquent d'éloigner le comportement de l'ouvrage de son comportement théorique.

Dans la littérature, de nombreux travaux présentent des instrumentations de quais en vue de la compréhension de leur comportement mécanique. Sur ces exemples, l'accent est mis sur la phase de chantier (Uff, J.F ${ }^{10}$, Blivet et al. ${ }^{1}$, Delattre et Mespoulhe ${ }^{4}$, Delattre et $a l .^{3}$, Gattermann et $a l .^{5}$, Rodatz et $a l^{7}$, Marten et $a l^{6}{ }^{6}$ ), et plus rarement sur le monitoring de l'ouvrage en service (Del Grosso et al. ${ }^{2}$ ). La stratégie utilisée dans les exemples cités consiste à traiter une section particulière de l'ouvrage sur laquelle on trouve généralement la concentration de la majeure partie des moyens de mesures. Elle se situe en général loin des bords et est sensée représenter le comportement d'une section courante. Cette stratégie est limitée car elle ne permet pas d'analyser des phénomènes variables sur toute la longueur d'un ouvrage ni d'évaluer les sources d'aléa. Toutefois, contrairement aux quais étudiés dans l'article présent, ces quais sont en général des parois disposants de plusieurs niveaux de tirants. Pour des questions de coût mais aussi d'influence sur l'organisation de chantier, il est alors nécessaire de se concentrer sur une problématique.

Dans le cadre de travaux sur l'approche probabiliste des indicateurs d'intégrité des structures maritimes et fluviales, l'équipe «Calcul des structures » du GeM a engagé une opération d'instrumentation et d'analyse de plusieurs quais situés dans l'estuaire de la Loire, en partenariat avec le Port Autonome de Nantes-Saint Nazaire - PANSN.

Cet article se focalise sur deux quais construits au début des années 2000 et identiques par leur conception. Il s'agit de quais sur pieux avec tirants d'ancrage. L'instrumentation présentée a pour la quantification des chargements horizontaux dus au remblai et au marnage. Après une description de ces ouvrages et de leur instrumentation, l'article présente une analyse comparée des variations à moyen et court terme des efforts dans les tirants d'ancrage puis s'intéresse à la distribution spatiale de ces efforts à des dates fixées.

\section{Description des quais sur pieux}

Les ouvrages étudiés, situés sur l'estuaire de la Loire, concernent l'extension du terminal à bois du Cheviré, le poste 4 (référencé quai C-4 par la suite) et l'extension du terminal à marchandises diverses et à conteneurs de Montoir, poste 4 (référencé quai TMDC-4 par la suite).

\subsection{Concept et exécution d'un quai sur pieux}

Le schéma de la figure 1 représente les éléments principaux d'un quai sur pieux : la plate-forme, les pieux, le talus, le remblai arrière, les ouvrages de soutènement, les tirants et les ancrages.

Les étapes de construction des deux quais diffèrent et ont été réalisées dans la direction amont-aval. Elles comprennent : des travaux de terrassement préliminaires, le battage des pieux, le battage du rideau de palplanches, le coffrage 
du mur d'arrière-quai, le bétonnage du quai et des pieux, la pose des éléments préfabriqués de la plate-forme, la mise en oeuvre et le compactage du remblai arrière-quai, la pose des tirants en deux tronçons (dans le cas du quai TMDC-4), la pose des tirants en un tronçon (dans le cas du quai C-4), le remblaiement, les travaux de terrassement finaux et l'installation des équipements.

La construction du quai TMDC-4 s'est étalée sur 16 mois, de novembre 2000 à février 2002 et celle du quai C-4, sur 14 mois d'octobre 2002 à décembre 2003.

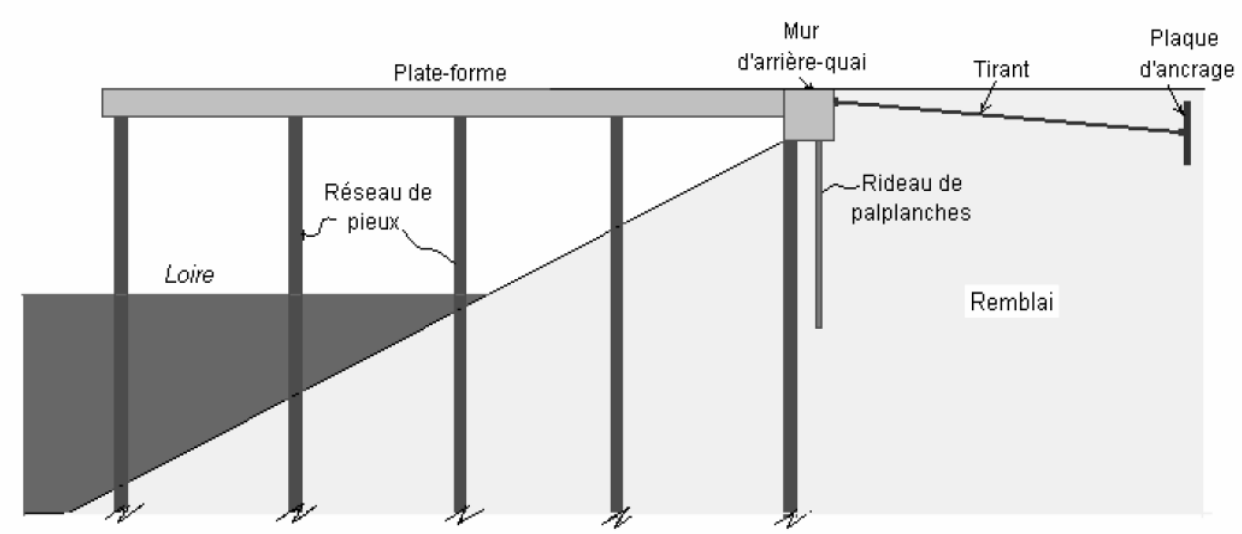

Figure 1 : Schéma d'un quai sur pieux (coupe transversale)

\subsection{Caractéristiques structurelles du quai TMDC-4}

Le quai TMDC-4 fait $256 \mathrm{~m}$ de longueur et $43 \mathrm{~m}$ de largeur. Il est situé sur la commune de Montoir, à deux kilomètres en aval du pont de Saint-Nazaire, sur la rive nord de l'embouchure de la Loire, en sortie d'estuaire. Ce terminal est prévu pour recevoir des navires de fort tonnage $(50.000 \mathrm{t}$ ), de type over-panamax, et d'un tirant d'eau maximal de $14 \mathrm{~m}$, ce qui est le cas des porte-conteneurs à l'usage desquels il est principalement destiné.

Ce quai est constitué d'une dalle de hauteur de $0,25 \mathrm{~m}$ en béton armé (B.A.), formée d'un réseau triangulaire de poutres en B.A. de $75 \mathrm{~cm}$ de hauteur, reposant sur un réseau d'environ 300 pieux métalliques battus remplis de béton d'une longueur d'environ $45 \mathrm{~m}$ et d'un diamètre extérieur selon les files de 711, $813 \mathrm{ou}$ $914 \mathrm{~mm}$. Le quai est adossé au remblai par l'intermédiaire d'un mur d'arrière-quai vertical en B.A. de 4,35 m de hauteur sur lequel s'appuie la dalle. Ce mur est soutenu par des pieux et coiffe un rideau parafouille de 5,2 $\mathrm{m}$ de hauteur composé de palplanches métalliques qui empêche la fuite des particules fines du remblai. Le quai est de plus ancré dans le remblai par 38 tirants d'ancrage passifs inclinés composés d'un cylindre d'acier de $85 \mathrm{~mm}$ de diamètre et de $20 \mathrm{~m}$ de longueur. Ces tirants sont fixés au mur d'arrière-quai par une rotule. Lors de la pose de ces tirants, s'ils ne sont pas suffisamment tendus, le jeu dans la rotule peut conduire à des efforts très faibles dans les tirants lors du déplacement du mur d'arrière-quai $\left(\right.$ Verdure $\left.^{11}\right)$. A l'autre extrémité, les tirants sont ancrés dans le sol par une plaque d'ancrage en B.A. dont les dimensions sont : H2,5 x L3,0 x 0,5 m. Le mur d'arrière-quai est traversé par des barbacanes qui se situent à une hauteur de 3,5 m 
dont la fonction est d'éviter une surcharge hydraulique lors des marées descendantes.

\subsection{Caractéristiques structurelles du quai C-4}

Pour l'extension du terminal à bois du PANSN, le quai C-4 est situé en aval du pont de Cheviré, à proximité de la ville de Nantes, sur la rive gauche de la Loire. Il s'agit d'un quai de $180 \mathrm{~m}$ de longueur et $34,50 \mathrm{~m}$ de largeur, prévu pour recevoir des navires de longueur maximale $225 \mathrm{~m}$ et d'un tirant d'eau de 9,10 m.

Le quai C-4 est construit sur un réseau de 198 pieux métalliques battus remplis de béton et ferraillés en partie haute, d'une longueur d'environ $33 \mathrm{~m}$ et d'un diamètre extérieur selon les files de 711, 762 ou $863 \mathrm{~mm}$. Sur chaque pieu est positionné un chapiteau destiné à centrer la charge transmise par le réseau poutre-dalle en tête du pieu. La dalle du quai en B.A. de $0,35 \mathrm{~m}$ de hauteur est composée de poutres en T en B.A. de 1,35 m de hauteur qui relient les pieux dans le sens transversal du quai. Le quai est ancré par 37 tirants d'ancrages cylindriques inclinés, en acier, de $75 \mathrm{~mm}$ de diamètre et de $15 \mathrm{~m}$ de longueur, situés derrière chaque file de pieux. Ces tirants sont fixés à une extrémité dans le mur d'arrière-quai (de 2,20 m de hauteur) au moyen d'une biellette, et à l'autre extrémité à une plaque d'ancrage carrée en B.A. de 2,6 x 2,6 x 0,5 m. A l'arrière du quai est fiché un rideau parafouilles de palplanches métalliques de $9 \mathrm{~m}$ de hauteur. Il est retenu en tête par le mur d'arrière-quai dont il est solidaire.

\section{Instrumentation pour le suivi du comportement sous chargement horizontal}

Une stratégie d'instrumentation originale a été réalisée (Schoefs et al. ${ }^{8}$ ). Elle vise à suivre le comportement global des quais, sous chargement horizontal, dans les 5 ans suivant leur construction afin de pouvoir mieux anticiper les évolutions dans le temps, et de faciliter la maintenance par une meilleure compréhension de leur fonctionnement en service. Elle constitue aussi le socle d'une mise à jour des variables et modèles qui devront être étendus pour le calcul en fiabilité des quais sous chargement de tempête. On cherche à instrumenter des composants de l'ouvrage ayant une sensibilité importante au chargement horizontal, difficilement accessibles après le chantier et à suivre le maximum de grandeurs influentes. Le chargement horizontal considéré est constitué des actions du remblai, de l'amarrage des navires et $\mathrm{du}$ vent sur les portiques. On a ainsi choisi d'instrumenter les tirants d'ancrages, et de suivre l'influence du niveau de la marée et de la nappe dans le remblai. L'originalité se trouve dans la volonté de disposer d'une forte proportion de tirants instrumentés $(25 \%)$ permettant de dégager les variations spatiales et de réaliser des modélisations probabilistes s'appuyant sur un traitement statistique.

Chaque quai a été instrumenté, à proximité du mur d'arrière-quai, sur douze tirants d'ancrage (régulièrement répartis sur la longueur de l'ouvrage) afin de suivre l'effort normal dans les tirants. Le choix d'une instrumentation située derrière l'arrière-quai permet d'éviter le traitement du frottement latéral sur les 
tirants, identifié comme phénomène important par Verdure ${ }^{11}$. Dans le cas du TMDC-4, la technologie utilisée a été celle des couples de capteurs à corde vibrante, fixés au moyen de brides spéciales (Schoefs et al. ${ }^{8}$ ). Dans le cas de C-4, on a utilisé une technique usuelle par jauges de déformation, directement collées à chaud sur les tirants. Dans les deux cas, ces systèmes préservent la protection anticorrosion des tirants. De plus, des mesures de hauteur de nappe d'eau dans le remblai ont été faites à l'aide de trois piézomètres sur le quai C-4 et deux sur le quai TMDC-4, implantés derrière le mur d'arrière-quai et reliés au poste d'acquisition qui centralise l'ensemble des données.

Les deux tirants situés à chaque extrémité du quai TMDC-4 ont été équipés de 3 couples de cordes vibrantes au lieu d'un pour l'étude de l'évolution de l'effort normal et du moment fléchissant le long du tirant, on pourra se reporter à $\left(\right.$ Verdure $\left.^{11}\right)$ pour plus de détails. De manière générale, les tirants instrumentés sont repérés et nommés par une lettre " $\mathrm{T}$ " et par leur abscisse longitudinale $\mathrm{x}$ en mètre, et par convention $\mathrm{x}=0$ à l'extrémité amont du quai.

Enfin, des marégraphes mesurent le niveau de la marée toutes les 5 minutes. Dans le cas du TMDC-4, les deux marégraphes du PANSN se situent à Donges $(4 \mathrm{~km}$ en amont) et à Saint-Nazaire ( $2 \mathrm{~km}$ en aval), ce qui permet d'extrapoler la valeur obtenue au niveau du quai. Pour C-4, le marégraphe du PANSN est situé à $1 \mathrm{~km}$ en aval du Pont de Cheviré. L'intérêt de ces mesures est que les surcotes sont intégrées.

\section{Analyse des mesures}

Les étapes sont la collecte des données fournies par le dispositif d'instrumentation, l'analyse des mesures brutes et le traitement afin de mettre en évidence des corrélations pertinentes, entre les efforts et la marée et entre efforts de tirants instrumentés voisins. L'acquisition de données est effectuée toutes les 30 minutes, ce qui permet de suivre les effets des marées et d'éviter un stockage trop important. Un prétraitement des données brutes de déformation est fait afin de calculer l'effort normal dans les tirants. Pour plus de détails on pourra se reporter à $\left(\right.$ Verdure $\left.^{11}\right)$ et $\left(\right.$ Yáñez-Godoy $\left.{ }^{13}\right)$. Les quais étant peu exploités durant ces mesures, et aucune relation entre la présence d'un navire et le niveau d'effort n'ayant pu être mise à jour, seuls les chargements de remblai sont analysés. Par ailleurs, il est important de souligner une perte de 20 à $25 \%$ de l'instrumentation, incluant diverses défaillances (fixation au support, rupture de câble, défaillance du capteur) : ce chiffre important est généralement constaté dans la littérature. Deux types de variations caractérisent les efforts dans les tirants :

- temporelles : variations à moyen terme, où se pose la question des niveaux d'efforts au cours d'un même mois et variations à court terme où l'on s'intéresse à l'amplitude des efforts durant une marée au cours d'une demi-journée ;

- spatiales : variations d'un tirant instrumenté à l'autre.

Dans les sections suivantes on analyse en détails ces deux types de variations. 


\subsection{Variations temporelles}

\subsubsection{Variations des efforts dans les tirants à moyen terme}

Les efforts moyens mensuels ont été choisis pour analyser l'évolution des efforts dans les tirants à moyen terme. Sur le quai TMDC-4, les mesures ont été recueillies tous les mois durant l'intervalle octobre 2002-octobre 2003. A partir de mai 2004 ce recueil a été fait de manière plus espacée. Toutes les mesures d'effort dans les tirants ont été prises 8 mois environ après qu'on ait installé les capteurs à corde vibrante, principalement pour des questions de phasage de chantier et de passage des gaines électriques. On distingue deux phases de vie de l'ouvrage : la phase 1, qui comprend l'intervalle octobre 2002 à juillet 2003, pendant laquelle les efforts évoluent peu et la phase 2, comprenant l'intervalle juillet 2003 à décembre 2005, pendant laquelle l'évolution des efforts varie en fonction du temps mais avec un comportement encore méconnu qui fait l'objet d'actuelles expertises.

A la différence du quai TMDC-4, sur le quai C-4 on a enregistré des mesures au cours du chantier. En effet, l'utilisation d'une mini-centrale portative a permis de réaliser les premières mesures lorsque l'ouvrage était en phase de construction, en août et septembre 2003. On a distingué ici une seule phase de vie de l'ouvrage dans l'intervalle de janvier 2004 à juin 2005, pendant laquelle les efforts évoluent selon la variabilité saisonnière. Depuis janvier 2004, la récupération de mesures a été faite de manière plus espacée. On notera des lacunes dans les données, dues à des coupures de gaine lors de travaux de finition et au délai de réparation.

\subsubsection{Influence de la marée sur les efforts dans les tirants}

On analyse maintenant plus en détails les variations d'effort à court terme c'est-àdire au cours d'une marée. De fait, un indicateur du fonctionnement des capteurs est le chargement périodique de la marée. Le type de marée observé sur l'estuaire de la Loire est semi-diurne (environ $12 \mathrm{~h}$ ). En plus, la Loire est peu sensible aux surcotes fluviales même si leur effet est perceptible sur le quai C-4. Afin d'isoler l'effet de la marée, on étudie les variations des efforts dans les tirants $\Delta \mathrm{F}=\mathrm{F}_{2}-\mathrm{F}_{1}$, correspondants aux variations des niveaux d'eau de la Loire $\Delta \mathrm{H}=\mathrm{H}_{2}-\mathrm{H}_{1}$, entre deux instants donnés $t_{2}$ et $t_{1}$. L'intervalle de temps sélectionné correspond à la périodicité des mesures (30 minutes). Des analyses exhaustives sont disponibles dans (Verdure et al. ${ }^{12}$ et Yáñez-Godoy ${ }^{13}$ ). A marée haute, l'effort dans les tirants est minimum, et les piézomètres indiquent alors des niveaux d'eau identiques du côté remblai à ceux fournis par les marégraphes côté Loire. Cette valeur minimale s'explique donc par un effet de déjaugeage dans le remblai. A marée basse, l'effort est maximum et des niveaux d'eau différents sont mesurés côté Loire et côté remblai, le niveau dans ce dernier étant le plus élevé. Sur les deux quais, on observe ainsi une sensibilité à la marée de tous les tirants, y compris ceux ayant un effort moyen très faible. Une forte corrélation linéaire entre les efforts dans les tirants et le niveau d'eau dans la Loire, mesuré par le marégraphe, est constatée. 
A titre d'exemple, on présente en figure $2 \mathrm{a}$ la variation de l'effort normal $\Delta \mathrm{F}$, par rapport à la variation du niveau de la Loire $\Delta \mathrm{H}$ lors d'une marée descendante pour les tirants du quai C-4. Certains tirants ne sont pas présentés en raison d'un dysfonctionnement, (voir introduction section 4). L'apparente non régularité du T180 par rapport aux autres tirants a déjà été identifiée lors des études à moyen terme. Ces corrélations quasi-linéaires sont observées sur les deux quais lors de marées descendantes et montantes. La sensibilité des tirants à la marée est directement liée à la raideur du complexe sol-tirant-plaque d'ancrage. Verdure a même montré $\left(\right.$ Verdure $^{11}$ ), que les tirants non sollicités, aux efforts presque nuls, peuvent avoir une sensibilité à la marée.

On a étudié dans (Yáñez-Godoy ${ }^{13}$ ) le comportement de chaque tirant instrumenté du quai C-4, au travers de l'évolution de $\Delta \mathrm{F} / \Delta \mathrm{H}$ sur une période de 4 mois, pour trois coefficients de marée (CMAR) : 42 pour les mortes-eaux, 69 pour les marées moyennes et 100 pour les vives eaux. La figure $2 \mathrm{~b}$ montre à titre d'exemple l'évolution de $\Delta \mathrm{F} / \Delta \mathrm{H}$, pour trois CMAR différents et pour des marées descendantes, correspondant au tirant T0 du quai C-4 au cours de la période janvier-octobre 2004. Les semaines 9 et 18 disposent de deux coefficients 100 . On définit un coefficient de sensibilité à la marée (CSM) égal à la variation en fonction du temps de $\Delta \mathrm{F} / \Delta \mathrm{H}$ :

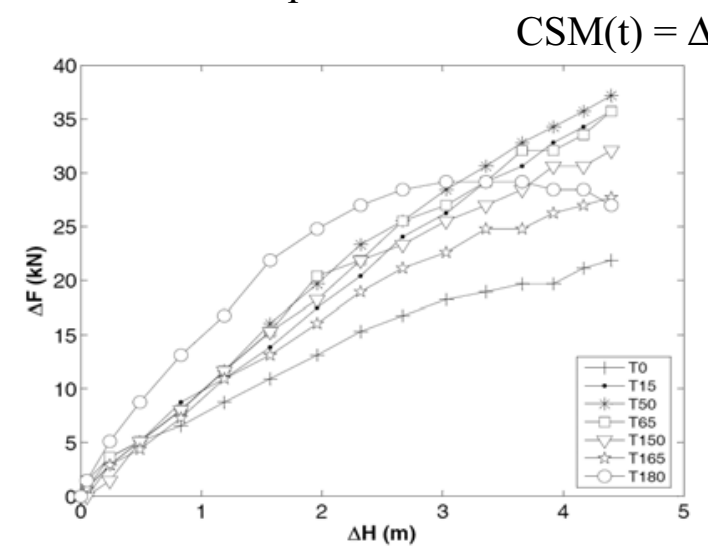

a) Marée descendante de $\mathrm{CMAR}=100$

Figure 2 : Variation de l'effort normal dans les tirants, du quai C-4, en fonction du niveau de la Loire

Les résultats obtenus ont montré que le CSM de chaque tirant, en présence de forts coefficients de marée, évoluait linéairement en fonction du temps ce qui met en évidence un phénomène de rigidification, ou comportement asymptotique, du complexe "sol-tirant-plaque d'ancrage ». Le quai TMDC-4 a fait l'objet d'une analyse identique avec les CMAR suivants : 42, 75 et 94. Les valeurs de ces coefficients diffèrent légèrement de celles sélectionnées pour $\mathrm{C} 4$ ce qui est dû à la quantité de valeurs disponibles sur la période d'observation.

\subsection{Variations spatiales des efforts}

Les variations temporelles ont permis d'identifier deux phénomènes caractérisant les évolutions du chargement depuis la mise en service de la chaîne d'acquisition : (1) dans le cas des évolutions à moyen terme, les efforts mesurés proviennent pour 
une partie du remblaiement et des conditions de mise en œuvre et pour une autre, des cycles saisonniers de la marée; (2) dans le cas des évolutions à court terme, on identifie une composante cyclique liée aux variations relatives de niveaux de nappes et du fleuve dues à la marée. Les deux phénomènes responsables des chargements horizontaux vont guider une analyse de la variation spatiale de l'effort le long des deux quais.

\subsubsection{Variation spatiale à moyen et court terme pour le quai TMDC-4}

Sur la figure 3a, on a représenté les efforts mesurés dans les tirants du TMDC-4 à différentes dates ainsi que le profil des valeurs moyennes. Sur les tirants T196 et T256, une erreur d'état 0 a certainement été réalisée et les valeurs obtenues ne sont pas présentées. Après traitement des données, on a attribué la borne inférieure mécaniquement admissible (valeur nulle), à tous les tirants ayant un effort légèrement négatif (cas des tirants T10, T17, T223 et de quelques valeurs du T136). On peut observer une grande variabilité d'un tirant à l'autre. La raideur du quai étant importante devant celle des pieux et des tirants d'ancrage, cette grande disparité des efforts dans les différents tirants ne peut être expliquée par une variation de raideur du quai. A titre de comparaison, on présente l'effort calculé résultant d'un équilibre limite en 2-D et égal à $370 \mathrm{kN}\left(\right.$ Verdure $\left.^{11}\right)$ pour une valeur du coefficient de poussée du remblai $\mathrm{K}_{\mathrm{a} 1}$ égale à 0,23 (Soubra et al. ${ }^{9}$ ). Cette approche très simplifiée ne peut rendre compte de la complexité de la situation observée et de résultats concernant une structure en service. Elle ne parvient cependant pas à envelopper l'ensemble des situations. La pré-charge imposée étant faible, elle peut venir d'une rotation de la plaque d'ancrage pendant le compactage mais aucune certitude ne peut être dégagée à ce stade de l'étude.

On a vu dans la section 4.1.2 que le comportement de chaque tirant, en présence de forts coefficients de marée, est représenté par un coefficient de sensibilité à la marée. On analyse ici la variation spatiale des efforts dans les tirants le long du quai TMDC-4 pour des marées montantes de CMAR $=94$. Sur la figure $3 \mathrm{~b}$ on a tracé un profil moyen représentatif de la variabilité spatiale au cours de la phase 1. La semaine 12 disposant de deux coefficients 94, on utilise la notation $12+$ On constate une variabilité d'un tirant à l'autre. On aurait pu prendre une marée descendante, les valeurs des sensibilités étant voisines.

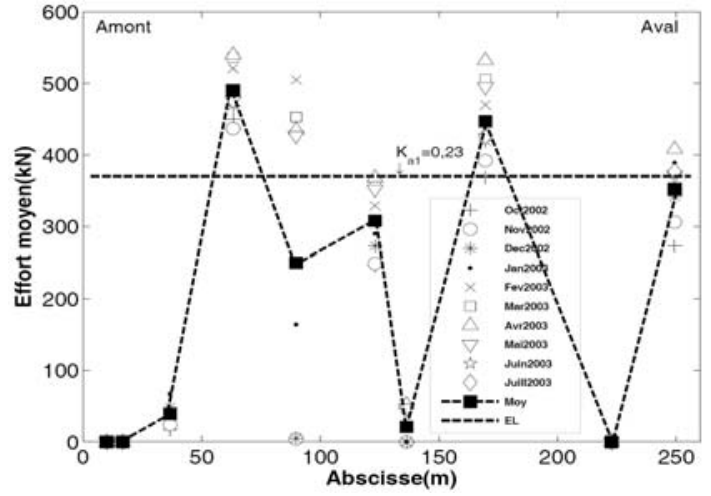

a) Moyen terme

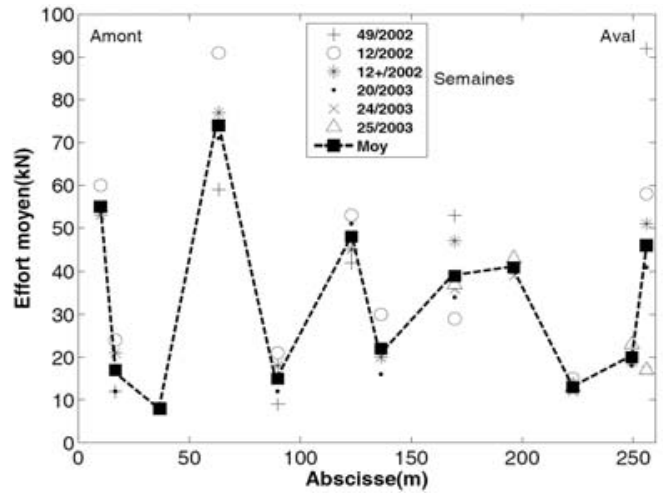

b) Court terme

Figure 3 : Evolution du profil d'efforts moyens dans les tirants du TMDC-4 (oct.2002 -juill. 2003) - données de Verdure ${ }^{11}$ complétées - 


\subsubsection{Variation spatiale à moyen et court terme pour le quai $\mathrm{C}-4$}

La figure 4a présente les efforts dans les tirants le long du quai C-4 d'une part sur la période de janvier 2004 à juin 2005 et d'autre part entre le 8/08/2003 et le 9/09/2003 en phase de chantier. On peut observer aussi une grande variabilité d'un tirant à l'autre. On affecte la valeur nulle aux tirants ayant des valeurs d'effort légèrement négatives, dans la plage de $10 \mathrm{kN}$ d'erreur (cas des tirants T165 et quelques valeurs du T180). On constate deux niveaux d'efforts distincts entre la zone amont (tirants T0, T15, T50 et T65) et la zone aval (tirants T80, T100, T150, T165 et T180). On note que les efforts dans les tirants présentés sur la note de calcul du quai C-4 sont de l'ordre de $691 \mathrm{kN}$ à l'état limite de service (ELS) et de $974 \mathrm{kN}$ à l'état limite ultime (ELU). Ces calculs ont considéré les actions combinées suivantes : effort d'accostage, effet du vent sur un portique et situation de tempête. La situation du quai pendant les mesures correspondait à un chargement du remblai seul (absence d'exploitation), et les valeurs à l'ELS mentionnées ci-dessus ne sont pas atteintes.

La figure $4 \mathrm{~b}$ présente la variation spatiale des efforts dans les tirants le long du quai C-4 lors de marées descendantes de CMAR $=100$, pour la période janvieroctobre 2004. Les variations d'un tirant à l'autre sont moins marquées pour ce quai C-4 que pour le TMDC-4. Seul T0 se distingue dû à un effet de bord.

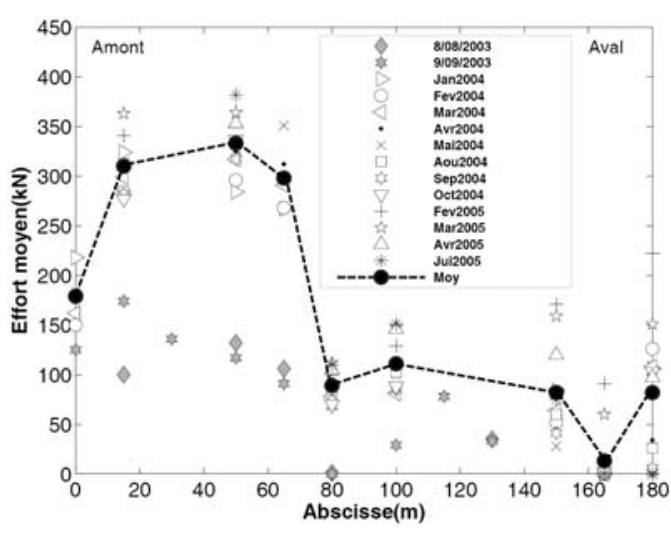

a) Moyen terme

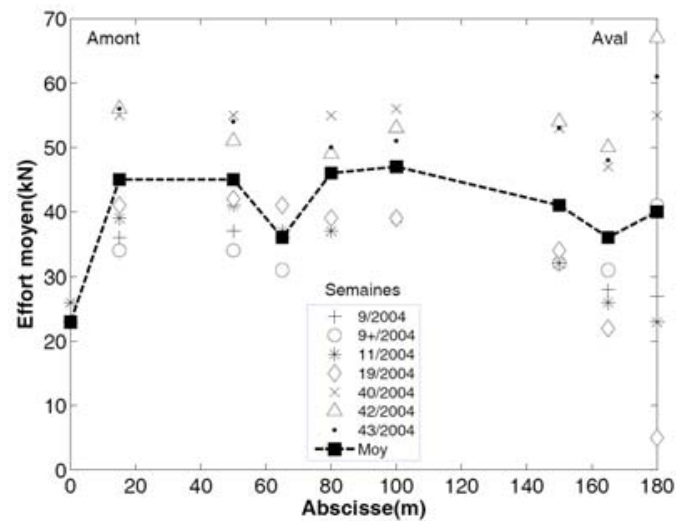

b) Court terme

Figure 4 : Evolution du profil d'efforts moyens mesurés dans les tirants du quai C-4, phase janvier 2004 à juin 2005 (pour l'évolution à moyen terme)

\section{$\underline{\text { 5. Conclusions }}$}

Cet article présente l'exploitation et la comparaison des données de mesures des efforts dans les tirants d'ancrage de deux quais sur pieux similaires situés en Loire et dans l'estuaire. Les variations temporelles à moyen terme des efforts dans les tirants étudiés pour les deux quais montrent une petite évolution de charge. Toutefois en effectuant l'analyse spatiale, nous avons trouvé une importante variabilité d'effort d'un tirant à l'autre. La moyenne et le maximum de l'effort mesuré dans tous les tirants est de $200 \mathrm{kN}$ et $540 \mathrm{kN}$ (TMDC-4) et de $150 \mathrm{kN}$ et $382 \mathrm{kN}(\mathrm{C}-4)$. Les variations à moyen terme des efforts mesurés dans les tirants proviennent pour une partie du remblaiement et des conditions de mise en œuvre 
et pour une autre des cycles saisonniers de la marée. Par ailleurs, l'étude de l'effet de la marée dans les tirants a montré comment le complexe sol-tirant-plaque d'ancrage se comporte pendant plusieurs mois. Cette étude a mis en évidence un phénomène de rigidification de ce complexe en présence de forts coefficients de marée. On notera enfin que les efforts mesurés en l'absence d'exploitation sont significatifs par rapport aux efforts calculés à l'ELS. Une analyse mécanique plus poussée devra permettre d'améliorer la modélisation et les risques de défaillance à l'ELS et à l'ELU.

\section{Références}

Blivet J.C., Bonafous P., Frank R., et Josseaume H., (1981), Comportement d'un quai en paroi moulée au port du Havre, Bulletin de liaison des Laboratoires des Ponts et Chaussées 113, France, May-June, 109-134.

2 Del Grosso A., Inaudi D. et Lanata F., (2000), Strain and displacement monitoring of a quay wall in the Port of Genoa by means of fibre optic sensors, 2nd ENPC European Conference, Champs-sur-Marne, France, 3-6 Juillet, on CD-Rom.

3 Delattre L., Duca V., Scherrer P. et Rivière P., (1999), Efforts d'ancrage du quai d'Osaka au Port Autonome du Havre, Proceedings of the 12th European Conference on Soil Mechanics and Geotechnical Engineering, Amsterdam, The Netherlands, 7-10 Juin, 713-718.

4 Delattre L., Mespoulhe L., (1999), Etude expérimentale du comportement du quai en eau profonde du port de Calais, Bulletin géotechnique GT65, Laboratoire Central des Ponts et Chaussées, France, avril, 193 p.

5 Gattermann J., Bergs T. et Rodatz W., (2001), Modified instrumentation and results of stress and deformation monitoring at the new quay wall construction - Container Terminal Altenwerder, Port of Hamburg, 8th International Conference on Structural Safety and Reliability, California, USA, 17-21 June, on CD-Rom.

6 Marten S., Delattre L., Nguyen et Burgeois E., (2004), The new deep water quay "Port 2000" of Le Havre, France : field monitoring and performance prediction, Numerical Models in Geomechanics - NUMOG IX, London, 565-571.

7 Rodatz W., Maybaum G. et Gattermann J., (1995), Pressure and deformation measurements at two retaining walls at the port of Hamburg, 4th International Symposium Field Measurements in Geomechanics (FMGM 95), Bergamo, Italy, 291299.

8 Schoefs F., Gerard B, Casari P. et Verdure L., (2004), Stratégie d'instrumentation pour la gestion optimisée des ouvrages portuaires, VIIIèmes Journées Nationales Génie Côtier-Génie Civil, Compiègne, France, 7-9 septembre, 513-520.

9 Soubra A.H., Macuh B., (2002), Active and passive earth pressure coefficients by a kinematical approach, Geotechnical engineering, vol. 155, $\mathrm{n}^{\circ}$ 2, Avril, 119-131.

10 Uff J.F., (1969), In situ measurements of earth pressure for a quay wall at Seaforth, Liverpool, Proceedings of the Conference organized by the British Geotechnical Society, London, England, 13-15 May, 229-239.

11 Verdure L., (2004), Cadre statistique du suivi en service des ouvrages de génie civil : application à un quai sur pieux, Thèse de doctorat, Université de Nantes, $232 \mathrm{p}$.

12 Verdure L., Casari P. et Wielgosz C., (2003), Joint use of instrumentation and probabilistic modelling applied to a container wharf, Proc. 9th Inter. Conf. On Applications of Statistics and Probability in civil engineering - ICASP 9, San Francisco, USA, on CD-Rom.

13 Yáñez-Godoy H., (2004), Identification d'indicateurs de performance d'un quai d'accostage en estuaire, Rapport de recherche DEA, Université de Nantes, 110 p. 